\title{
Multimodal Imaging of Optic Nerve Drusen in a 10-year Hyperopic Child
}

\author{
Sehnaz Ozcaliskan and Ozgur Artunay \\ Department of Ophthalmology, University of Health Sciences, Beyoglu Eye Training and Research Hospital Istanbul, Turkey
}

\begin{abstract}
Optic nerve drusen (OND) are abnormal calcified acellular deposits located within the prelaminar portion of the optic nerve. They are usually asymptomatic and detected incidentally during fundoscopic examination. They have a clinical significance due to their appearance which mimicks true papilledema, if the drusen are buried. Misdiagnosis of an OND as papilledema may cause unnecessary and invasive interventions, like lumbar puncture for elevated intracranial pressure. We present a case of buried OND in a 10year hyperopic girl who was referred to our clinic with the complaint of headache and swollen optic discs bilaterally. After diagnostic tests, including B-scan ultrasonography (USG) and enhanced depth imaging optic coherence tomography (EDI OCT), she was diagnosed with bilateral OND.
\end{abstract}

Key Words: Children, Drusen, Imaging, Optic Nerve, Papilledema.

How to cite this article: Ozcaliskan S, Artunay O. Multimodal Imaging of Optic Nerve Drusen in a 10-year Hyperopic Child. J Coll Physicians Surg Pak 2020; 30(05):541-542. DOI: https://doi.org/10.29271/jcpsp.2020.05.541.

\section{INTRODUCTION}

Optic nerve drusen (OND) have a reported prevalence in children of $0.37 \%$ to $1 \%{ }^{1,2}$ They are typically buried in children and become more superficial and visible as the children's age increases. Although the exact pathogenesis is not well known, impairment of axonal transport and ganglion cell death are thought to play a role in the development of OND. ${ }^{3}$ OND are usually asymptomatic, but they have a clinical significance due to their appearance, which mimicks true papilledema if the drusen are buried. Misdiagnosis of an OND as papilledema may result in unnecessary and invasive interventions like lumbar puncture for elevated intracranial pressure. B-scan ultrasonography (USG) is a reliable non-invasive test for diagnosis of OND. Recently, the role of enhanced depth imaging optic coherencetomography (EDIOCT) in the differential diagnosis of OND and papilledema also has been reported in literature. In this case, we aimed to illustrate and discuss imaging findings of a buried OND in a hyperopic girl.

\section{CASE REPORT}

A 10-year girl was referred to our clinic with the complaint of headache and bilateral optic disc swelling. She had a history of car accident and blunt force trauma to the head three weeks ago and was reviewed by a pediatrician. Her systemic evaluation, including neurologic examination and neuroimaging, did not reveal any significant finding. In her ophthalmologic evaluation, best corrected visual acuity was 20/32 with +8.5 spherical diopters (D) in both eyes. No color vision deficit could be elicited.

Correspondence to: Sehnaz Ozcaliskan, Beyoglu Eye

Training and Research Hospital, 34421, Beyoglu, Istanbul,

Turkey

E-mail:drsehnaz@yahoo.com

Received: July 22, 2019; Revised: July 23, 2019;

Accepted: July 28, 2019

DOI: https://doi.org/10.29271/jcpsp.2020.05.541
Direct and consensual pupillary light reflexes were normal with no relative afferent pupillary defect. Biomicroscopic examination of the anterior segment was unremarkable and intraocular pressure were normal in her both eyes. The axial lengths were 19.94 and $19.90 \mathrm{~mm}$ for her right and left eyes, respectively. Fundoscopic examination revealed bilateral swollen optic discs with indistint and irreguler margins. There were no hyperemia, hemorrhages and congestion (Figure 1).

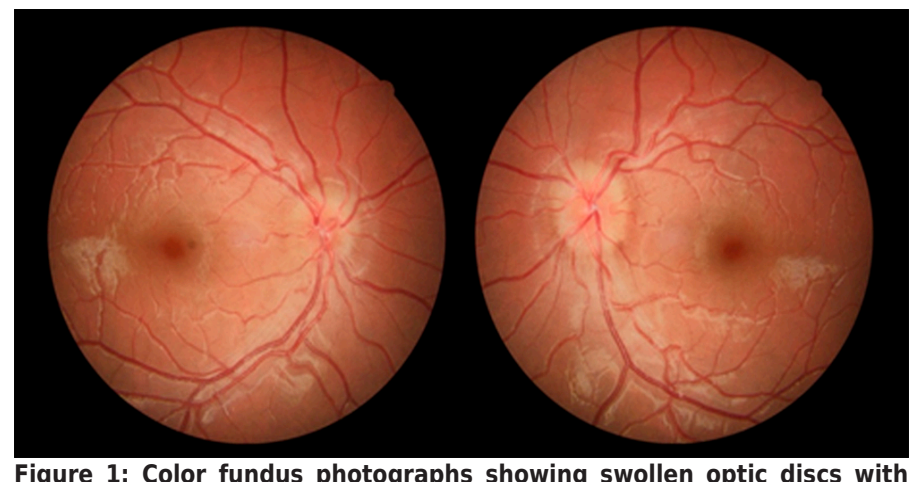

Figure 1: Color fundus photographs showing swollen optic discs with indistint and irregular margins.

On B-scan USG, an ovoid hyperechogenic lesion was revealed on the optic nerves bilaterally. Fundus autofluorescence (FAF) imaging and retinal nerve fiber layer thickness analysis with spectral domain OCT was normal.

EDI OCT of the optic nerve heads showed an amorphous material with hyperreflective spots bilaterally. It also revealed that the outer plexiform layer band was diverging anteriorly and the retinal pigment epitelium and Bruch's membrane was deflexing backwards (Figure 2).

Her previous orbital computerised tomography (CT) scans, performed on the day of the car accident, revealed a bright spot at the optic nerve head, which was also compatible with the diagnosis of the OND(Figure 3). 


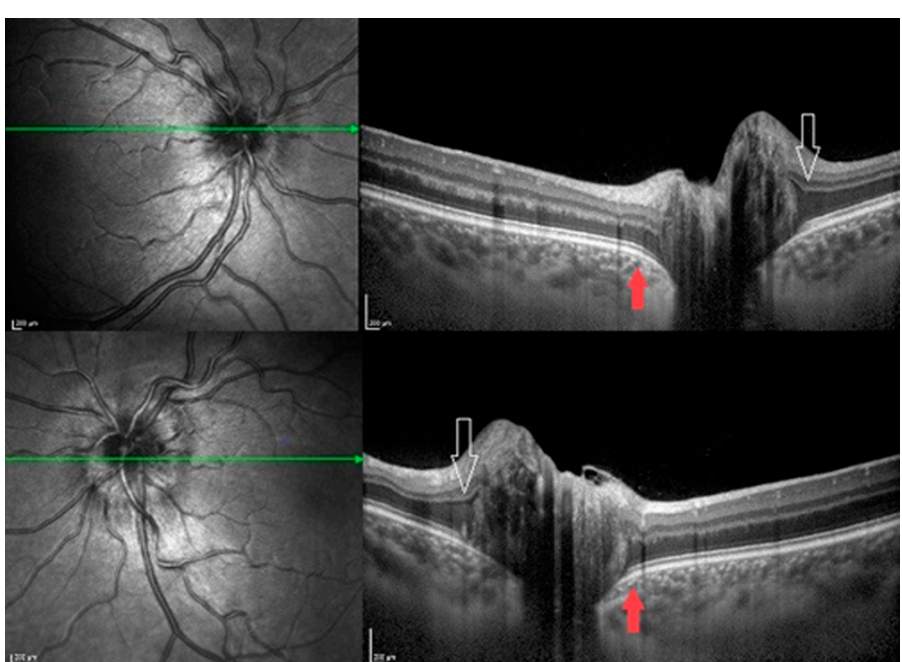

Figure 2: Enhanced depth imaging OCT of the optic nerve head showing an amorphous material with hyperreflective spots. Outer plexiform layer band is diverging anteriorly (white arrow) and the retina pigment epitelium and bruch membraneare deflexing backwards (red arrow).

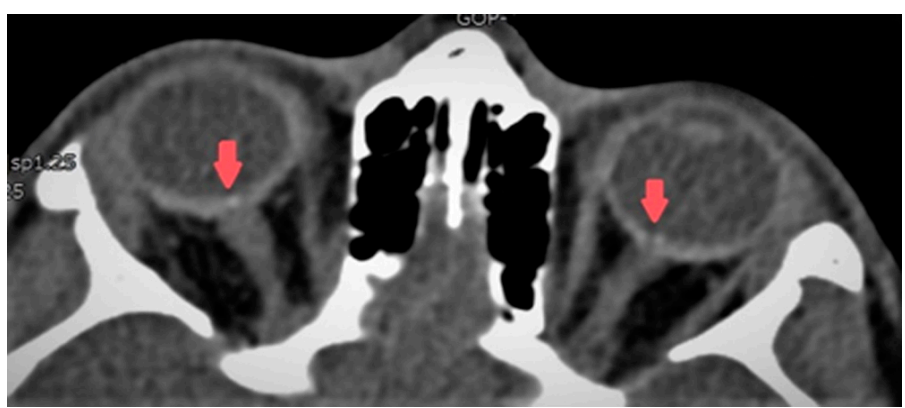

Figure 3: Orbital CT scan showing a bright signal at junction of the posterior globe and optic nerve (red arrow).

\section{DISCUSSION}

OND are abnormal calcified acellular deposits located within the prelaminar portion of the optic nerve. ${ }^{4}$ They are usually asymptomatic and detected incidentally during fundoscopic examination. Population studies have reported a prevalence of $0.34 \%-2 \%$ with bilateral asymmetric distribution (75\%).

The pathogenesis of OND still remains unclear. They are caused by the alteration in axonal metabolism with slowed axoplasmic flow of ganglion cells. ${ }^{3}$ A small scleral canal that compresses the optic nerve may compromise the axonal transport, leading to ganglion cell death, extrusion and calcification of mitochondria. Previous studies had shown that shorter and hyperopic eyes are associated with OND. In a recent study, the mean scleral canal diameter in eyes with OND was below $25 \%$ percentile in $92 \%$ of children with OND. ${ }^{2}$

The fundoscopic appearence of the OND gradually alters throughout lifetime. In early life, OND are buried by neural and vascular structures. Buried drusen elevate the optic disc and blur the margins. As the patient gets older, drusen calcify, and become more superficial and visible. They protrude from the edge of the optic disc and cup, particularly on the inferior nasal side and appear as refractile whiteyellow bodies on the surface of the optic nerve. ${ }^{8}$ While superficial drusen are easy to identify, buried drusen are difficult to differentiate from true papilledema, particularly when they are bilateral. But unlike papilledema, optic disc hyperemia, peripapillary hemorrhage, venous obstruction and exudates are not the common findings. Spon- taneous venous pulsations and filling-in of the physiological cup are often present in OND. Because OND are more frequently non-calcified and buried, it is more difficultto detect these in children.

Several imaging methods, including FAF, orbital CT, B-scan USG and OCT have been used to diagnose OND. B-scan USG had been accepted as the gold standard in the diagnosis of drusen and found to be superior to CT scan. ${ }^{9}$ Since the diagnostic ability of B-scan USG is related to the calcification rate of the tissue, non-calcified OND may not be detected by USG. In FAF, OND appear as a round to irregular autofluorescent structures. In our case, FAF did not show any findings. It is hypothesised that FAF does not reliably detect buried drusen, possibly because of attenuation from overlying tissue. ${ }^{10}$ EDI OCT can define the size and structure of the OND more accurately than B-scan USG and non-EDI OCT. It has a higher OND detection rate and also provides better visualisation of the deeper posterior structures. ${ }^{10}$

Clinical distinction between OND and papilledema is crucial, particularly in the pediatric population. With a comprehensive ophthalmologic examination, including evaluation of refractive errors and imaging of the disc, OND may be properly diagnosed; and unnecessary invasive procedures for papilledema can be avoided.

\section{PATIENT'S CONSENT:}

Written informed consent was obtained from the patient for publication of this case report accompanying images.

\section{CONFLICT OF INTEREST:}

The authors have declared no conflict of interest.

\section{AUTHORS' CONTRIBUTION:}

SO, OA: Contributed to the design and data collection, literature review and writing of the manuscript.

\section{REFERENCES}

1. Erkkila H. Clinical appearance of optic disc drusen in childhood. Albrecht Von Graefes Arch Klin Exp Ophthalmol 1975; 193:1-18.

2. Malmqvist L, Li XQ, Eckmann CL, Skovgaard AM, Olsen EM, Larsen M, et al. Optic disc drusen in children: The Copenhagen child cohort 2000 eye study.J Neuroophthalmol 2018; 38:140-6.

3. Tso MO. Pathology and pathogenesis of drusen of the optic nervehead. Ophthalmology 1981; 88:1066-80.

4. Chang MY, Pineles SL. Optic disk drusen in children. Surv Ophthalmol 2016; 61:745-58.

5. Friedman AH, Gartner S, Modi SS. Drusen of the optic disc. A retrospective study in cadaver eyes. Br J Ophthalmol 1975; 59:413-21.

6. Auw-Haedrich C, Staubach F, Witschel H. Optic disk drusen. Surv Ophthalmol 2002; 47:515-32.

7. Ghassibi MP, Chien JL, Abumasmah RK, Liebmann JM, Ritch R, Park SC. Optic nerve head drusen prevalence and associated factors in clinically normal subjects measured using optical coherence tomography. Ophthalmology 2017; 124:320-5.

8. Wilkins JM, Pomeranz HD. Visual manifestations of visible and buried optic disc drusen. J Neuroophthalmol 2004; 24:125-9.

9. Kurz-Levin MM, Landau K. A comparison of imaging techniques for diagnosing drusen of the optic nerve head. Arch Ophthalmol 1999; 117:1045-9.

10. Merchant KY, Su D, Park SC, Qayum S, Banik R, Liebmann JM, et al. Enhanced depth imaging optical coherence tomography of optic nerve head drusen. Ophthalmology 2013; 120: 1409-14. 